Research Article

Cellular, Molecular and Developmental Genetics

\title{
Isosorbide mononitrate promotes angiogenesis in embryonic development of zebrafish
}

\author{
Hui $\mathrm{Lv}^{1}$, Bo Liu ${ }^{2}$ and Yongwen $\mathrm{Qin}^{3}$ \\ ${ }^{1}$ The Second Affiliated Hospital of ShanXi Medical University, Department of Cardiovascular Disease, \\ Taiyuan, Shanxi, 030001, China. \\ ${ }^{2}$ Xinhua Hospital Affiliated To Shanghai Jiaotong University School Of Medicine, Department of \\ Cardiovascular Disease, Shanghai 200092, China. \\ ${ }^{3}$ The Second Military Medical University, Department of Cardiovascular Disease, Shanghai 200433, China.
}

\begin{abstract}
Coronary heart disease (CHD) is a leading cause of death worldwide, and angiogenesis plays important roles in CHD. Thus, in the present study, the angiogenic efficacy of four common cardiovascular medicines (aspirin, pravastatin, metoprolol and isosorbide mononitrate (ISMN)) was determined by the number and length of zebrafish intersegmental vessels (ISVs) after immersing zebrafish embryos in different medicines. Results showed that ISMN significantly increased the length and number of ISVs. ISMN is a long-acting nitrate ester drug. It has been used as a vasodilator to dilate arteries and veins to reduce the cardiac preload and postload. However, the effect of ISMN on angiogenesis remains unclear. Thus, by in vitro experiments, the angiogenic mechanism of ISMN was evaluated through detecting the viability and proliferation of human umbilical vein endothelial cells (HUVECs) and the expression of angiogenesis-related genes and miRNAs. Results indicated that ISMN could increase the viability and proliferation of HUVECs by decreasing apoptosis, and elevated the expressions of vedf, kdrl, pdgfr in zebrafish embryos. Furthermore, the expressions of miR-126, miR-130a and miR-210 were also regulated in ISMN-treated HUVECs. In conclusion, ISMN could promote angiogenesis in zebrafish embryos and HUVECs, implying ISMN may be a potential therapeutic in treating angiogenesis-related diseases.
\end{abstract}

Keywords: Isosorbide mononitrate, angiogenesis, coronary heart disease, zebrafish, miRNA.

Received: July 13, 2019; Accepted: May 28, 2020.

\section{Introduction}

Coronary heart disease (CHD) is still the major cause of death in the world even if the past 20 years witnessed a dramatic decline (Colquhoun et al., 2000; Capewell and O'Flaherty, 2008; Jones and Greene, 2013). In recent decades, research found that angiogenesis plays central roles in the pathological process of CHD (Zhang et al., 2018). Therapeutic angiogenesis is an alternative approach to augment the innate myocardial angiogenesis and formation of collateral circulations following ischemia for the no-option patients (Grass et al., 2006). Angiogenesis was modulated by a subset of signaling pathways including vascular endothelial growth factor (Vegf)-Vegfr, Tie-angiopoietin, transforming growth factor (TGF)-beta, platelet-derived growth factor (Pdgf), and integrins (Warren and Iruela, 2010). Meanwhile, in recent years, miRNAs have been reported to regulate various stages of angiogenesis. MiRNAs are 21-23 nucleotides

Send correspondence to Yongwen Qin. The Second Military Medical University, Department of Cardiovascular Diseases, Shanghai 200433, China. E-mail: 18135370680@163.com. long, single stranded noncoding RNA molecules. MiRNA's capacity to target genes within a signaling pathway makes them promising target for anti-angiogenesis drugs (Tiwari et al., 2018). It is demonstrated that a few specific miRNAs, such as miR-126, miR-210, 221 and 222, could regulate angiogenesis process (Poliseno et al., 2006; Chen and Gorski, 2008; Urbich et al., 2008).

Previously, studies demonstrated that statins, a kind of cardioprotective drug, could suppress the growth of cancer cells through its antiangiogenic activities (Wang et al., 2010), and induce angiogenesis in stroke and ischemic heart disease (Elewa et al., 2012). Moreover, other types of cardiovascular medicines, such as antiplatelet drug, $\beta_{1}$ receptor blocker and nitrates, also have potentials to regulate angiogenesis (Ulu et al., 2009; Su et al., 2014; Rammos, 2015). But, the efficacy and mechanism of these cardioprotective drugs on the therapeutic angiogenesis remain unclear. Thus, in the present study, we selected pravastatin (a statin), aspirin (an antiplatelet drug), metoprolol (a $\beta_{1}$ receptor blocker) and ISMN (a nitrate) to test their angiogenic abilities.

To investigate the roles of cardiovascular medicines in angiogenesis and identify the underlying mechanisms, in 
this study, $\operatorname{Tg}(f l k 1: e G F P)$ zebrafish embryo angiogenesis model and human umbilical vein endothelial cells (HUVECs) were employed. The angiogenic efficacy of different types of cardioprotective drugs was determined by the number and length of zebrafish intersegmental vessels (ISVs). Here, we preliminarily noticed that ISMN significantly enhanced the ISV growth. As ISMN belongs to nitrates, we further investigated whether nitrates can promote angiogenesis. Hence, different kind of nitrates, including nitroglycerin (NTG), isosorbide dinitrate (ISDN) and ISMN were further used to test their roles in angiogenesis. Among these nitrates, ISMN increased the length and number of ISV significantly, thus, the angiogenic mechanism of ISMN was further evaluated by detecting apoptosis in HUVECs and the expressions of vedf, $k d r l, f l t-4$, flil $a$ and $p d g f r$ in zebrafish embryos. Thereafter, the expressions of miR-126, miR130a, miR-210, miR-221 and miR-222 were also measured.

\section{Material and Methods}

\section{Zebrafish embryo collection and drug administration}

$\operatorname{Tg}(f l k: e G F P)$ zebrafish embryos, in which vascular endothelial cells are fluorescently stained with the enhanced green fluorescent protein (eGFP), were generated by natural pairwise mating and were maintained at $28.5^{\circ} \mathrm{C}$ in embryo water as described by Westerfield (Cross et al., 2003). Different types of drugs were dissolved in $0.5 \%$ Dimethyl sulfoxide (DMSO) to form different concentration of solutions. The concentration of drugs was based on the blood drug level tested in human being. Zebrafish embryos were arrayed in 24-well plate and incubated with $2 \mathrm{~mL}$ solution containing various concentration per well at $28.5^{\circ} \mathrm{C}$ for $24 \mathrm{~h}$. Embryos incubated in DMSO (0.5\%) were served as control. Each trial was performed with three times of repetition at least, with 25 embryos each group. The length of ISVs was measured by the software provided by LEICA company which can calculate point to point distances.

\section{Cell line and cell culture}

Human Umbilical Vein Cells (HUVEC) (ATCC, VA, USA) were maintained in DMEM medium (Invitrogen, CA, USA) supplemented with $2 \%$ fetal bovine serum, $100 \mathrm{U} / \mathrm{mL}$ penicillin and $100 \mu \mathrm{g} / \mathrm{mL}$ streptomycin at $37{ }^{\circ} \mathrm{C}$ with $5 \%$ $\mathrm{CO}_{2}$ (Lam et al., 2008).

\section{RNA extraction}

Total RNA from zebrafish embryos at $0,6,12,24 \mathrm{hpf}$ was isolated using Trizol-Reagent according to the manufacture's protocol. Ten zebrafish embryos or about $1 \times 10^{5}$ cells were harvested from each group. The quantity and quality of RNA were estimated by spectrophotometer (Qiagen, Germany). The ratio of $\mathrm{OD}_{260} / \mathrm{OD}_{280}$ should be above 1.8.

\section{Quantitative real-time PCR (qRT-PCR) analysis}

The total RNA extracted from zebrafish embryos or cells were reverse transcribed to cDNA by using PrimeScript RT reagent Kits (TaKaRa, Dalian, China) with special stem-loop primer for miRNA and oligo-dT or random primer for mRNA. qRT-PCR was performed on a Rotor-Gene instrument (Qiagen, Germany) using SYBR Green. The primers used in the amplification were listed in the Table S1 and Table S2. The housekeeping genes U6 and gapdh were applied as internal standards for miRNAs and mRNA, respectively. The cycling program was set as follows: $94^{\circ} \mathrm{C}$, $15 \mathrm{~s}, 58^{\circ} \mathrm{C}, 15 \mathrm{~s}, 72^{\circ} \mathrm{C}, 20 \mathrm{~s}, 40$ cycles. Relative abundance was calculated by the delta-delta $\mathrm{Ct}$ method.

\section{MTT assay for cell viability}

The assessment of cell vitality was performed by MTT assay. Briefly, HUVECs were seeded into 96-well cell culture plates at an initial density of $1 \times 10^{4}$ cells/well. Following a $24 \mathrm{~h}$ treatment of $4 \mathrm{ng} / \mathrm{mL}, 20 \mathrm{ng} / \mathrm{mL}, 0.1 \mu \mathrm{g} / \mathrm{mL}, 0.5$ $\mu \mathrm{g} / \mathrm{mL}$ and $5 \mu \mathrm{g} / \mathrm{mL}$ ISMN, $20 \mu \mathrm{L}$ of a $5 \mathrm{mg} / \mathrm{mL}$ solution in PBS of the MTT substrate (Sigma-Aldrich, MO, USA) was added and incubated for up to $4 \mathrm{~h}$. The resulting blue-brown formazan precipitate formed was solubilized by DMSO. A curve of cell vitality was constructed by measuring cell growth with a microplate reader at $490 \mathrm{~nm}$.

\section{Cell proliferation}

The surface of each well of the 48-well cell culture dish was coated with $200 \mu \mathrm{L}$ Matrigel Matrix (BD Biosciences). Then, the culture dish was incubated for $2 \mathrm{~h}$ at $37^{\circ} \mathrm{C}$ to solidify the Matrigel. HUVECs were spread at $2 \times 10^{4}$ cells $/$ well. They were then treated with ISMN at $0.05 \mu \mathrm{g} / \mathrm{mL}$, $0.5 \mu \mathrm{g} / \mathrm{mL}$ and $5 \mu \mathrm{g} / \mathrm{mL}$ respectively for $6 \mathrm{~h}$, and the images of cells were captured by a CCD camera (AxioCam HC, Carl Zeiss, Thornwood, NY). Eight fields were randomly selected and the cell number was counted by Image $\mathrm{J}$ software (National Institutes of Health; http://rsbweb.nih.gov/ij/).

\section{Flow cytometry assay for cell apoptosis}

After treated with $5 \mu \mathrm{g} / \mathrm{mL}$ ISMN for $24 \mathrm{~h}$, adherent HUVECs were released by trypsinization. The cell apoptosis was investigated by Annexin V: FITC kit (Roche, Basel, Switzerland) based on the manufacturer's specifications. The samples were analyzed by a Becton Dickinson FACS Aria cell sorter.

\section{Statistical analysis}

The data was presented as mean \pm SD. Data was analyzed using SPSS software 16.0 (SPSS Inc., Chicago, IL). Statistical significance was assessed by one-way ANOVA. $P$ $<0.05$ were considered as statistically significant.

\section{Results}

\section{ISMN accelerated blood vessel formation}

To figure out the effects of cardiovascular medicines on angiogenesis, we first examined the vessel formation of zebrafish embryos. $T g(f l k 1: e G F P)$ zebrafish embryos $(\mathrm{n}=$ 25 each group) were treated with 4 types of drugs, including aspirin (antiplatelet drug), pravastatin (statins), metoprolol 
( $\beta_{1}$ receptor blocker) and ISMN (nitrates). There was no obvious difference in the development of dorsal aorta among the 4 treatment groups at 12 hours post fertilization (hpf) and 24 hpf (data not shown). However, the number of intersegmental vessels (ISVs) was significantly increased in the ISMN group compared with that in the other 3 groups ( $p$ $<0.05$ ) (Figures 1A and 1B). Furthermore, as shown in Figure $1 \mathrm{C}$, the length of ISVs in ISMN-treated embryos was significantly elevated compared with that in the control group $(p<0.05)$, whereas, other drugs showed no significant effect on the ISVs growth.

\section{ISMN promoted angiogenesis}

According to the above findings, as a nitrate, ISMN could promote ISVs growth. Moreover, in a previous study, researchers found that nitric oxide donors could be successfully used for the treatment of developed angiogenesis-inhibitor-induced hypertension (Kruzliak et al., 2013). Thus, we speculate that some nitrates may also play a role in angiogenesis. Therefore, we further explored the impacts of various common nitrates cardioprotective drugs on the growth of ISVs. Nitroglycerin (NTG), isosorbide dinitrate (ISDN) and ISMN were used to treat zebrafish embryos thrice, twice and once a day independently according to their pharmacokinetics in human. Interestingly, compared with the control embryos, the lengths and numbers of ISVs were only significantly increased in ISMN-treated group at $24 \mathrm{hpf}(p<0.05)$ (Figure 2).
(A)

Control

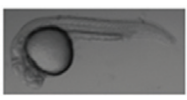

Aspirin
$10 \mu \mathrm{g} / \mathrm{mL}$

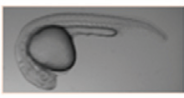

Pravastatin $8 \mu \mathrm{g} / \mathrm{mL}$

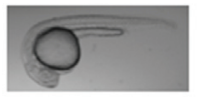

Metoprolol

$5 \mu \mathrm{g} / \mathrm{mL}$

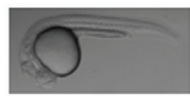

ISMN $500 \mu \mathrm{g} / \mathrm{mL}$

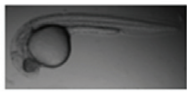

(B)

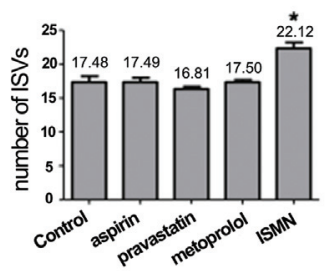

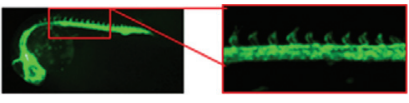
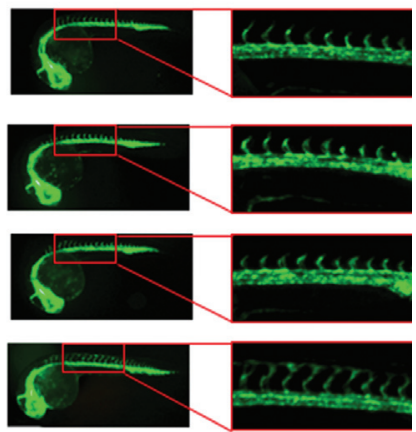

(C)

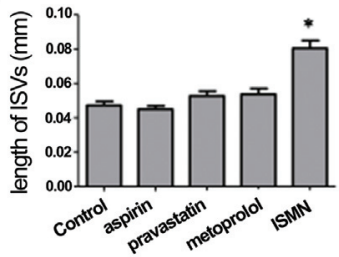

Figure 1 - Screening results of cardiovascular drugs with angiogenic ability using $T g(f l k: E G F P)$ zebrafish. (A) Fluorescence images of $24 \mathrm{hpf}$ zebrafish embryos treated with cardiovascular drugs for $24 \mathrm{~h}$. (B) The number of ISVs in zebrafish embryos. (C) The length of ISVs in zebrafish embryos. Data were expressed as mean $\pm \mathrm{SD}(\mathrm{n}=3){ }^{*} p<0.05$.
(A)

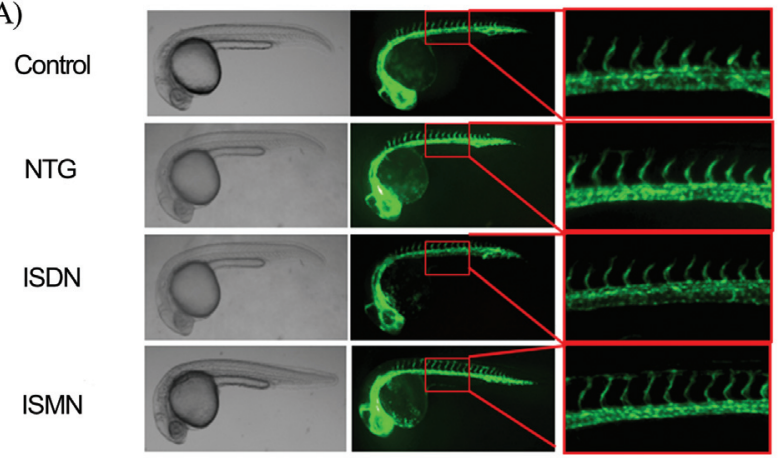

(B)

(C)
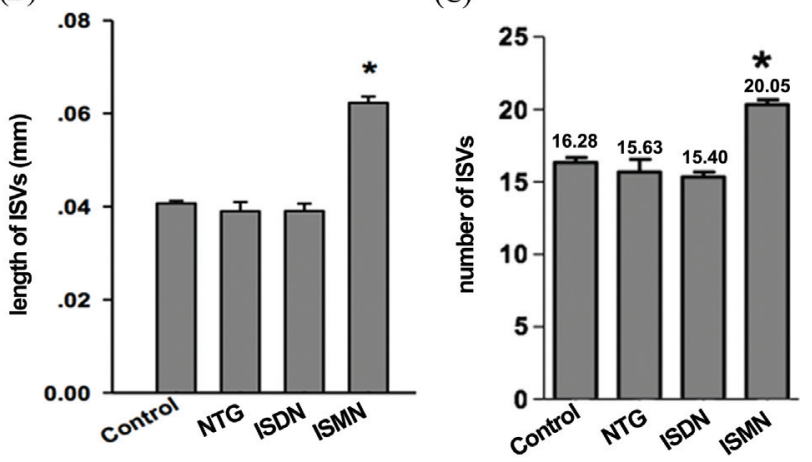

Figure 2 - Effects of nitrates on angiogenesis of ISVs. (A) Fluorescence images of zebrafish embryos at $24 \mathrm{hpf}$ treated with DMSO (control) and different nitrates. (B) The number of ISVs in zebrafish embryos treated with nitrates. (C) The length of ISVs in zebrafish embryos treated with nitrates. Data were expressed as mean $\pm \mathrm{SD}(\mathrm{n}=3) . * p<0.05$.

ISMN treatment up-regulated expressions of vegf-a, $k d r l$, and pdgfr in zebrafish embryos

In order to investigate the molecular mechanisms of ISMN-induced angiogenesis, expression levels of selected genes involved in angiogenesis process were detected at a series of incubation time using qRT-PCR. Vegf-a, a major regulator for angiogenesis, could bind and activate Vegfr2 (Kdrl) in zebrafish (Schuermann et al., 2014). Figure 3 shows that ISMN could significantly increase mRNA levels of vegf- $a$ and $k d r l$ at $12 \mathrm{hpf}$ and $24 \mathrm{hpf}(p<0.05)$. No significant changes were observed in vegfr 3 (flt4) mRNA expression. Flila, one factor of ETS domain gene, also links with angiogenesis (Brown et al., 2000). Figure 4A indicates that ISMN could not alter the flila expression level significantly. Moreover, $p d g f r$, which can affect vascular development, was up-regulated in ISMN treated embryos at $24 \mathrm{hpf}(p<$ 0.05 ) (Figure 4B). Hence, these results suggest that the up-regulation of vegf-a, $k d r l$, and $p d g f r$ expressions by ISMN may contribute to the pro-angiogenesis in zebrafish embryos.

\section{ISMN increased HUVECS viability and proliferation and decreased HUVECS apoptosis}

The effects of ISMN at $4 \mathrm{ng} / \mathrm{mL}, 20 \mathrm{ng} / \mathrm{mL}, 0.1$ $\mu \mathrm{g} / \mathrm{mL}, 0.5 \mu \mathrm{g} / \mathrm{mL}$, and $5 \mu \mathrm{g} / \mathrm{mL}$ on cell viability of HUVEC 

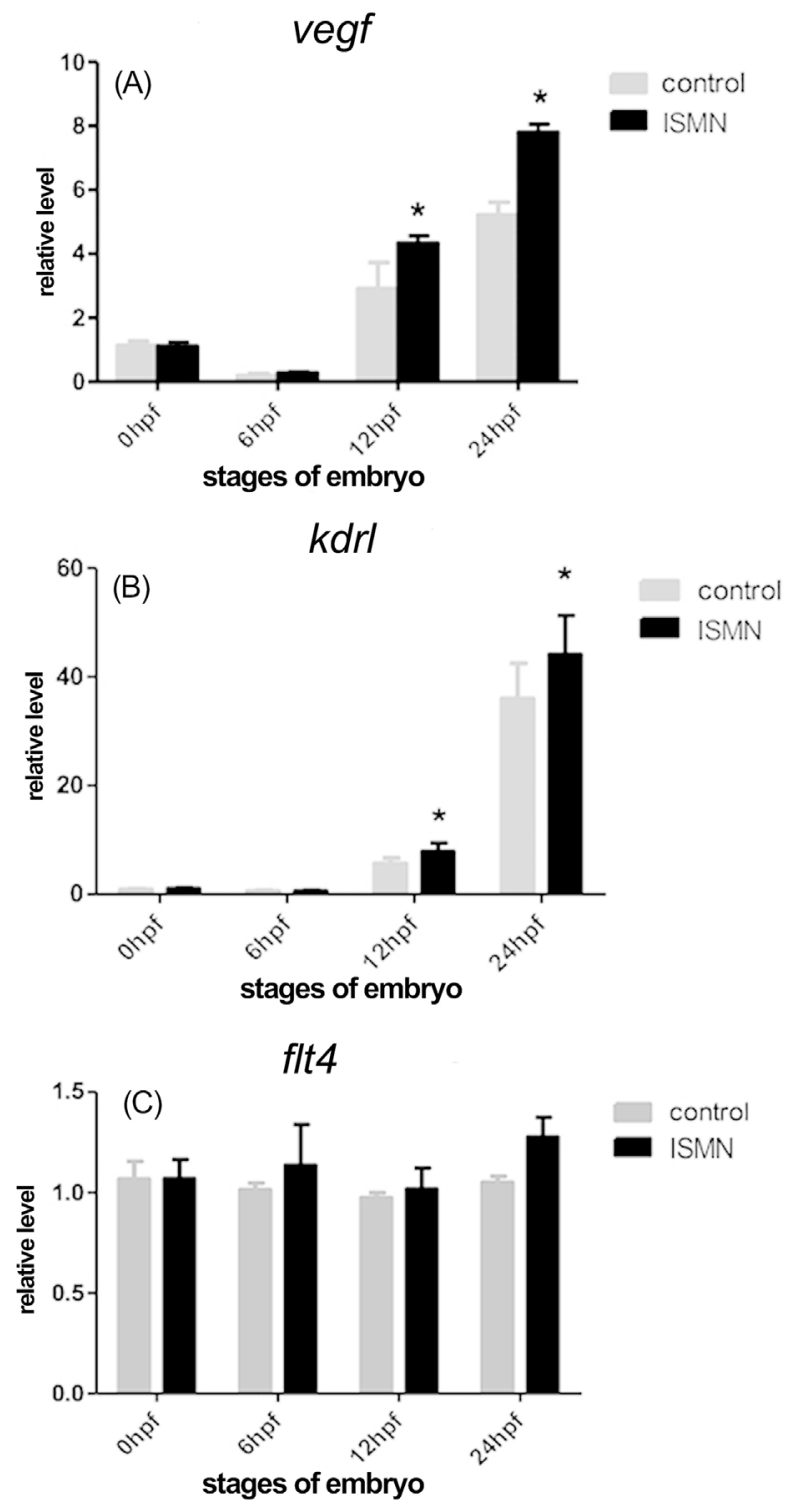

Figure 3 - Analysis of target genes involved in VEGF signaling pathways in ISMN-treated zebrafish embryos. (A) Relative expression of vegf- $a$. (B) Relative expression of vegfr-2 ( $k d r l)$. (C) Relative expression of vegfr-3 $(f l t 4)$. Data were expressed as mean $\pm \mathrm{SD}(\mathrm{n}=3) . * p<0.05$.

were assessed by MTT assay. As shown in Figure 5A, we observed that ISMN increased cell viability in a dose-dependent manner, and $5 \mu \mathrm{g} / \mathrm{mL}$ ISMN significantly elevated HUVECs viability $(p<0.05)$. Then, the proliferation of HUVECs was also investigated. Based on Figure 5B, after treating HUVECs with ISMN at $0.05 \mu \mathrm{g} / \mathrm{mL}, 0.5 \mu \mathrm{g} / \mathrm{mL}$ and $5 \mu \mathrm{g} / \mathrm{mL}$, the cell numbers were significantly increased $(p<$ $0.01)$ compared with the control group. To determine if the increased cell viability and proliferation were induced by ISMN was due to less apoptosis, Annexin-V staining was utilized (Figure 6). The average number of non-viable cells and AnnexinV $\mathrm{V}^{+} / \mathrm{PI}^{+}$cells in ISMN-treated HUVECs was decreased compared to the control ones $(24.02 \%$ vs $5.42 \%$;
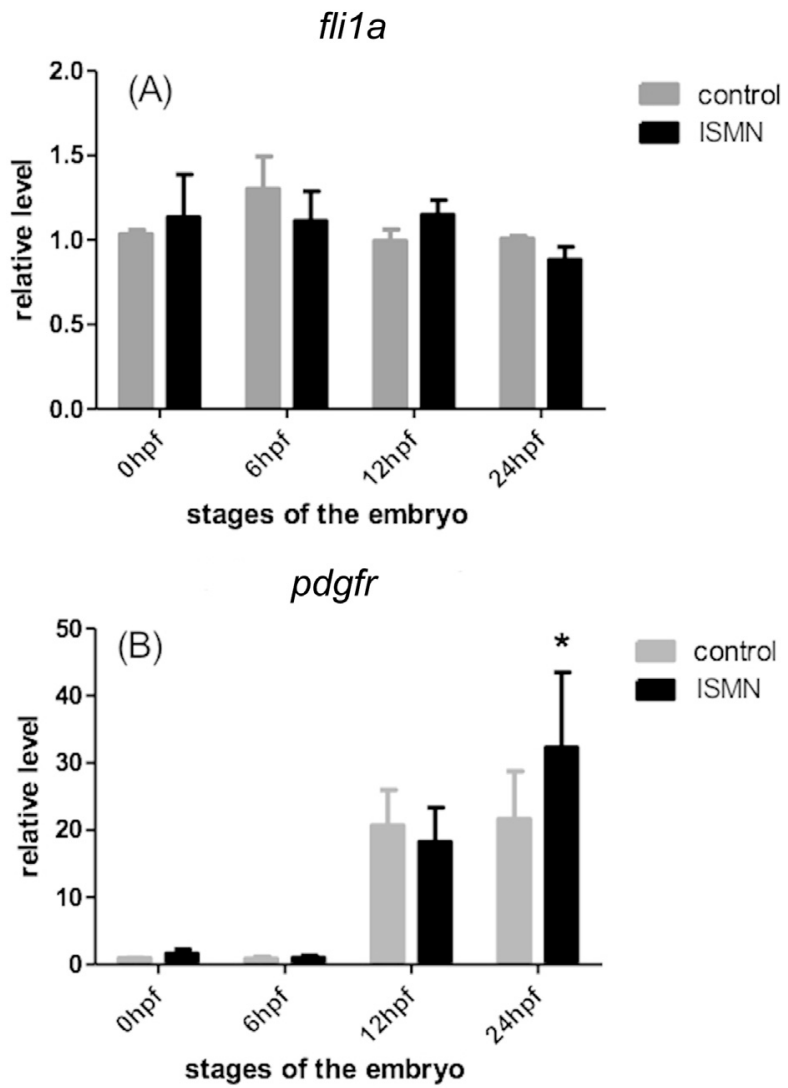

Figure 4 - Expressions of flila and $p d g f r$ in zebrafish embryos treated with ISMN for $24 \mathrm{~h}$. (A) flila expression. (B) $p d g f r$ expression. Data were expressed as mean $\pm \mathrm{SD}(\mathrm{n}=3) . * p<0.05$.

$14.32 \%$ vs $2.95 \%$ ), indicating that ISMN could decrease apoptosis in HUVECs.

\section{Expression of miRNAs involved in angiogenesis in HUVECS induced by ISMN}

ISMN at different concentrations was used to treat HUVECs to analyze the expression of miRNAs involved in angiogenesis. As shown in Figure 7, the expression of miR126 was significantly down-regulated by ISMN at all the tested concentrations $(p<0.05)$. Besides, $5 \mu \mathrm{g} / \mathrm{mL}$ ISMN could significantly enhanced the expressions of miR-130a and miR-210 $(p<0.05)$. Nevertheless, no significant changes in the expressions of miRNA-221 and miRNA-222 were observed (Figure 7). Altogether, these results suggested that the increased expressions of miR-130a and -210, and the decreased expression of miR-126 induced by ISMN promoted angiogenesis of HUVECs.

\section{Discussion}

Angiogenesis therapy was once considered as an alternative to traditional revascularization in no-option patients, but recently, it has opened unprecedented opportunities for CHD treatment (Emanueli and Madeddu, 2006). The effects of pro-angiogenic therapy against ischemic diseases have been validated in various experiments (Cao, 2010). Beside, 


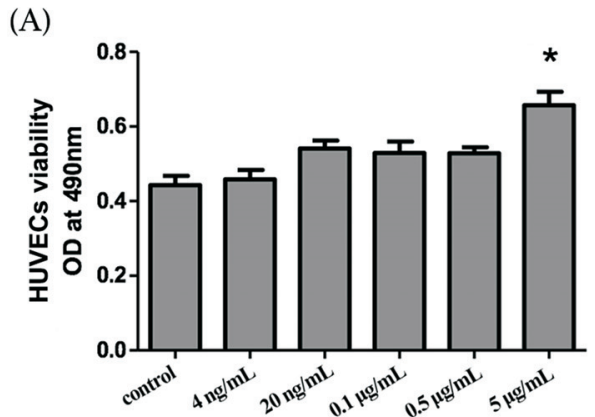

(B)
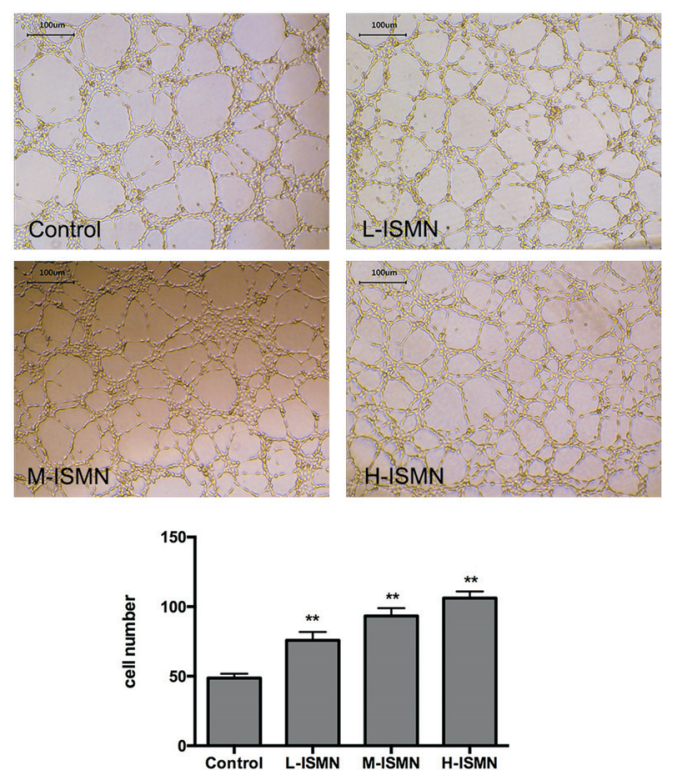

Figure 5 - Effects of ISMN on HUVECs viability and proliferation. (A) HUVECs viability determined by MTT. (B) HUVECs proliferation. L-ISMN, $0.05 \mu \mathrm{g} / \mathrm{mL}$; M-ISMN, $0.5 \mu \mathrm{g} / \mathrm{mL}$; H-ISMN, $5 \mu \mathrm{g} / \mathrm{mL}$. DMEM treatment was used as control. Data were expressed as mean $\pm \mathrm{SD}(\mathrm{n}=3)$. $* p<0.05$.

some cardiovascular medicines also show potentials to affect angiogenesis (Ulu et al., 2009; Su et al., 2014). However, the efficacy and mechanisms of these medicines in angiogenesis are still unclear.

In the current study, we investigated the angiogenic effects of different cardiovascular drugs, including aspirin, pravastatin, metoprolol and ISMN. ISMN, one of the most frequently used compounds for CHD treatment, is an organic nitrate vasodilator that can reduce myocardial oxygen demand and increase oxygen supply by vasodilating capacitance veins and coronary arteries (Stockis et al., 2002). Here, we found that only ISMN could increase the number and the length of ISVs in zebrafish embryos, indicating the potential angiogenic ability of ISMN. Then, the above findings promoted us to explore whether other nitrates also have angiogenic abilities. It is reported that $24 \mathrm{hpf}$ is an important time for ISVs formation (Ellertsdottir et al., 2010). By using the clinically most relevant organic nitrates (NTG, ISDN and ISMN), we noticed that only ISMN showed an angiogenic effect at $24 \mathrm{hpf}$ in zebrafish embroys, which may due to the

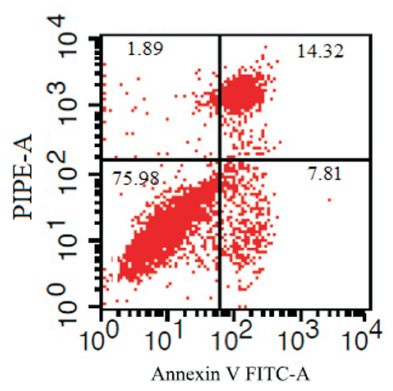

Control

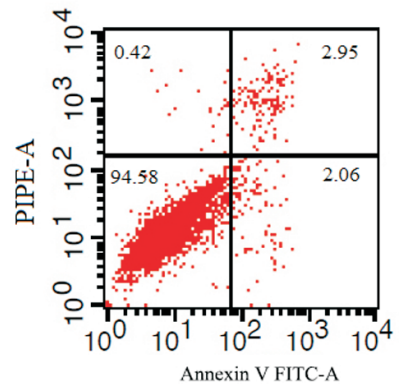

ISMN
Figure 6 - Effects of ISMN on HUVEC apoptosis by Annexin V-FITC/PI flow cytometry. Q1 (annexin V-/PI+) - cells with features of necrosis; Q2 (annexin $\mathrm{V}+/ \mathrm{PI}+$ ) - cells with features of late apoptosis; Q3 (annexin V-/PI-) - viable cells; Q4 (annexin V+/PI-) - cells with features of early apoptosis.

different chemical structures of these nitrates (Csont and Ferdinandy, 2005).

Organic nitrates can exert their biological effects via the release of nitric oxide (NO) (Cao, 2010). NO can induce angiogenesis through regulating VEGF (Salvolini et al., 2010). Vegf and Vegfrs are major contributors to zebrafish vascular development. In the Vegf family, Vegf and Vegfr-2 (Kdrl) play important roles in the angiogenesis and formation of collateral vessels (Yla-Herttuala et al., 2007; Liu and Patient, 2008). In the present study, ISMN significantly increased the mRNA level of vegf and vegfr $2(k d r l)$, which implies that the angiogenic effects of ISMN can be modulated by VEGF expression. Besides, Vegfr-3 (flt4) is mainly expressed in lymphatic endothelial cells (EC) (Lymboussaki et al., 1998), but the flt4 expression was not altered significantly by ISMN. Moreover, ISMN treatment also significantly up-regulated the expression of $p d g f r$, which is required for zebrafish angiogenesis process (Wiens et al., 2010). Although flila is also essential for angiogenesis (Liu and Patient, 2008), ISMN did not change flila transcription level significantly. Therefore, the up-regulation of vegf-a, $k d r l$, and $p d g f r$ expressions by ISMN may contribute to the pro-angiogenesis in zebrafish embryos.

Furthermore, by taking advantage of HUVECs, we further examined the mechanisms of ISMN on angiogenesis. The in vitro data indicates that ISMN increased HUVECs viability in a dose-dependent manner. Meanwhile, ISMN also enhanced the proliferation and decreased the apoptosis of HUVECs. Altogether, the angiogenic effect of ISMN may be achieved through the regulation of endothelial cell viability.

MicroRNAs are a class of conserved non-coding small RNAs, which can cause post-transcriptional inhibition of gene expression by targeting the 3' UTRs of mRNAs (Bushati and Cohen, 2007). Much evidence indicates that miRNAs are key regulators in angiogenesis and endothelial function (Wang and Olson, 2009). MiR-126 is the only miRNA of EC-specific expression and miR-126 level is decreased when VEGF signaling pathway is activated (Zhu et al., 2011). In the current study, we found that miR-126 expression was significantly down-regulated in HUVECs exposed to ISMN. Moreover, our data also showed that ISMN 

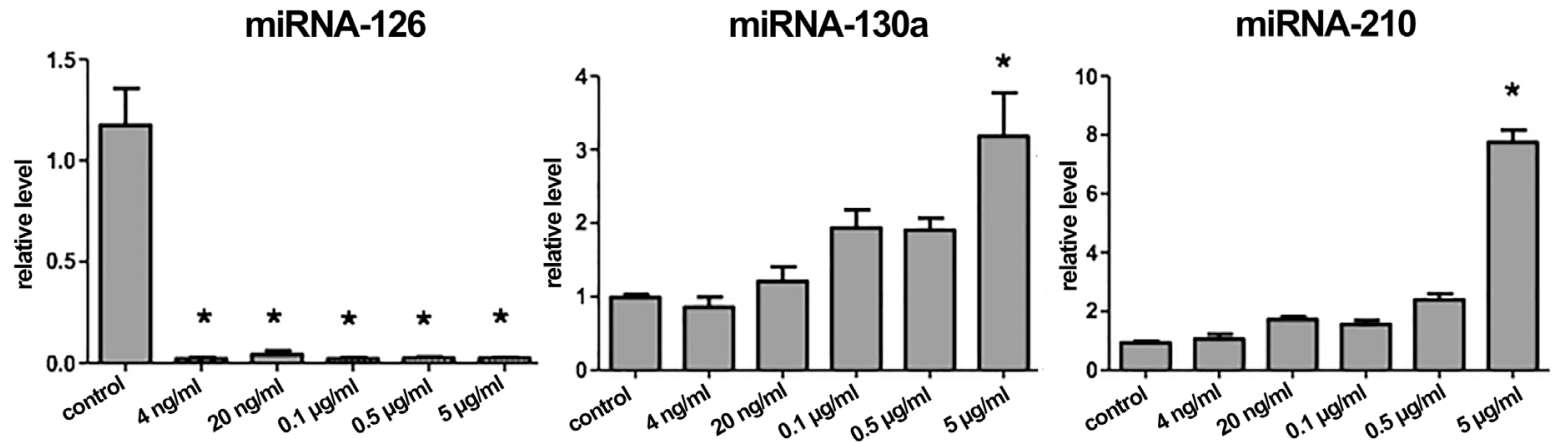

miRNA-221

miRNA-222
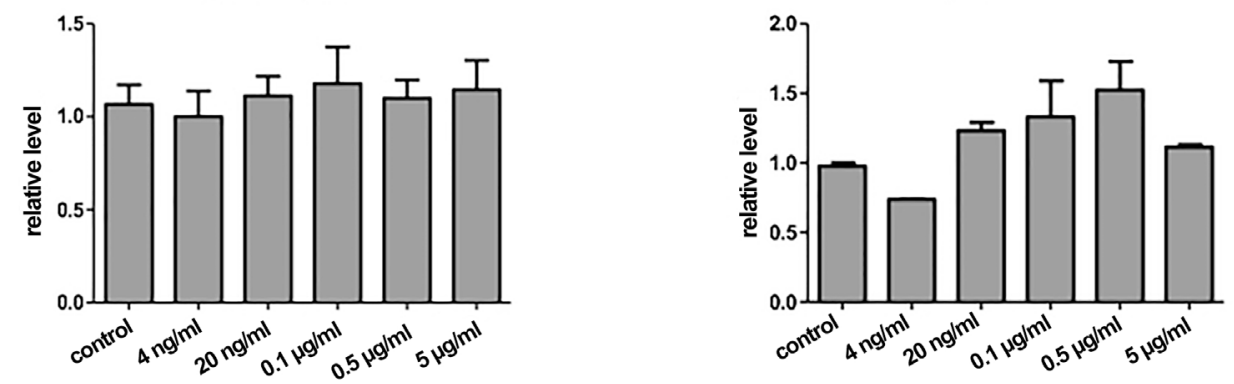

Figure 7 - Effects of different levels of ISMN on miRNAs expressions in HUVECs after $24 \mathrm{~h}$ treatment. Data were expressed as mean $\pm \operatorname{SD}(\mathrm{n}=3) . * p<$ 0.05 .

treatment significantly up-regulated the expression of miR$130 \mathrm{a}$, which can down-regulate the anti-angiogenic protein and promote angiogenesis (Chen and Gorski, 2008). Additionally, miR-210, which can enhance the formation of capillary-like structures (Tiwari et al., 2018), was shown to up-regulate in the present experiment. Among the miRNAs with higher expression in HUVECs, miR-221 and miR-222 were reported to resist angiogenesis (Poliseno et al., 2006), but in our study, miR-221 and -222 had no obvious responses to ISMN treatment. Taken together, the increased expressions of miR-130a and -210, and the decreased expression of miR-126 induced by ISMN may play a role in angiogenesis.

In conclusion, our study revealed that ISMN could promote angiogenesis in embryonic development of zebrafish via regulating vegf-a, $k d r l$, and $p d g f r$. Moreover, by using HUVECs, we found that the decreased apoptosis, downregulated miR-126 level and up-regulated levels of miR-210 as well as miR-130a may also contributed to angiogenesis. These findings deepen our understanding of the angiogenic ability of cardiovascular drugs in treating CHD. However, limitations exist in the present work. It remains to know whether inhibition and overexpression of the angiogenic genes could influence angiogenesis. Moreover, clinical trials should also be designed for therapeutic evaluation of the pro-angiogenic therapy with ISMN. Thus, future studies should be employed to further clarify the specific angiogenic mechanisms mediated by ISMN.

\section{Acknowledgement}

This work was supported by the Department of Cardiovascular Diseases of Changhai Hospital in Shanghai, China.

\section{Conflicts of Interest}

The authors declare no conflict of interest

\section{Author Contributions}

YWQ designed the experiment; HL wrote the manuscript; BL analyzed the data. All authors read and approved the final version.

\section{References}

Brown LA, Rodaway ARF, Schilling TF, Jowett T, Ingham PW, Patient RK and Sharrocks A (2000) Insights into early vasculogenesis revealed by expression of the ETS-domain transcription factor Fli-1 in wild-type and mutant zebrafish embryos. Mech Dev 90:237-252.

Bushati N and Cohen SM (2007) microRNA functions. Annu Rev Cell Dev Biol 23:175-205.

Cao Y (2010) Angiogenesis: What can it offer for future medicine? Exp Cell Res 316:1304-1308.

Capewell S and O'Flaherty M (2008) What explains declining coronary mortality? Lessons and warnings.Heart 94:1105-1108.

Chen Y and Gorski DH (2008) Regulation of angiogenesis through a microRNA (miR-130a) that downregulates antiangiogenic homeobox genes GAX and HOXA5. Blood 111:1217-1226.

Colquhoun D, Tonkin A, Schrapnel B and Sormerset S (2000) Dietary misconceptions and missed opportunities to help pa- 
tients lower coronary heart disease (CHD) risk. Atherosclerosis 151:A113.

Cross LM, Cook MA, Lin S, Chen JN and Rubinstein AL (2003) Rapid analysis of angiogenesis drugs in a live fluorescent zebrafish assay. Arterioscler Thromb Vasc Biol 23:911-912.

Csont T and Ferdinandy P (2005) Cardioprotective effects of glyceryl trinitrate: Beyond vascular nitrate tolerance. Pharmacol Ther 105:57-68.

Elewa HF, Elremessy AB, Somanath PR and Fagan SC (2012) Diverse effects of statins on angiogenesis: New therapeutic avenues. Pharmacotherapy 30:169-176.

Ellertsdottir E, Lenard A, Blum Y, Krudewig A, Herwig L, Affolter $M$ and Belting HG (2010) Vascular morphogenesis in the zebrafish embryo. Dev Biol 341:56-65.

Emanueli C and Madeddu P (2006) Therapeutic angiogenesis: Translating experimental concepts to medically relevant goals. Vascul Pharmacol 45:334-339.

Grass TM, Lurie DI and Coffin JD (2006) Transitional angiogenesis and vascular remodeling during coronary angiogenesis in response to myocardial infarction. Acta Histochem 108:293-302.

Jones DS and Greene JA (2013) The decline and rise of coronary heart disease: understanding public health catastrophism. Am J Public Health 103:1207-1218.

Kruzliak P, Kovacova G and Pechanova O (2013) Therapeutic potential of nitric oxide donors in the prevention and treatment of angiogenesis-inhibitor-induced hypertension. Angiogenesis 16:289-295.

Lam HW, Lin HC, Lao SC, Gao JL, Hong SJ, Leong CW, Yue PYK, Kwan YW, Leung AYH, Wang YT et al. (2008) The angiogenic effects of Angelica sinensis extract on HUVEC in vitro and zebrafish in vivo. J Cell Biochem 103:195-211.

Liu F and Patient R (2008) Genome-wide analysis of the zebrafish ets family identifies three genes required for hemangioblast differentiation or angiogenesis. Circ Res 103:1147-1154.

Lymboussaki A, Partanen TA, Olofsson B, Thomas-Crusells J, Fletcher CD, de Waal RM, Kaipainen A and Alitalo K (1998) Expression of the vascular endothelial growth factor $\mathrm{C}$ receptor VEGFR-3 in lymphatic endothelium of the skin and in vascular tumors. Am J Pathol 153:395-403.

Poliseno L, Tuccoli A, Mariani L, Evangelista M, Citti L, Woods K, Mercatanti A, Hammond S and Rainaldi G (2006) MicroRNAs modulate the angiogenic properties of HUVECs. Blood 108:3068-3071.

Rammos C (2015) Potential of dietary nitrate in angiogenesis. World J Cardiol 7:652.

Salvolini E, Lucarini G, Zizzi A, Orciani M, Di Benedette G and Di Primio R (2010) Human skin-derived mesenchymal stem cells as a source of VEGF and nitric oxide. Arch Dermatol Res 302:367-74.

Schuermann A, Helker CSM and Herzog W (2014) Angiogenesis in zebrafish. Semin Cell Dev Biol 31:106-114.

Stockis A, De Bruyn S, Deroubaix X, Jeanbaptiste B, Lebacq E, Nollevaux F, Poli G and Acerbi D (2002) Pharmacokinetic profile of a new controlled-release isosorbide-5-mononitrate
$60 \mathrm{mg}$ scored tablet (monoket multitab). Eur J Pharm Biopharm 53:49-56.

Su BB, Chen JH, Shi H, Chen QQ and Wan J (2014) Aspirin may modify tumor microenvironment via antiplatelet effect. Med Hypotheses 83:148-150.

Tiwari A, Mukherjee B and Dixit M (2018) MicroRNA key to angiogenesis regulation: MiRNA biology and therapy. Curr Cancer Drug Tar 18:266-277.

Ulu N, Henning RH, Goris M, Schoemaker RG and van Glist WH (2009) Effects of ivabradine and metoprolol on cardiac angiogenesis and endothelial dysfunction in rats with heart failure. J Cardiovasc Pharm 53:9-17.

Urbich C, Kuehbacher A and Dimmeler S (2008) Role of micrornas in vascular diseases, inflammation, and angiogenesis. Cardiovasc Res 79:581-588.

Wang C, Tao W, Wang Y, Bikow J, Lu B, Keating A, Verma S, Parker TG, Han R and Wen XY (2010) Rosuvastatin, identified from a zebrafish chemical genetic screen for antiangiogenic compounds, suppresses the growth of prostate cancer. Eur Urol 58:418-426.

Wang S and Olson EN (2009) AngiomiRs-Key regulators of angiogenesis. Curr Opin Genet Dev 19:205-211.

Warren CM and Iruela-Arispe L (2010) Signaling circuitry in vascular morphogenesis. Curr Opin Hematol 17:213-218.

Wiens KM, Lee HL, Shimada H, Metcalf AE, Chao MY and Lien CL (2010) Platelet-derived growth factor receptor beta is critical for zebrafish intersegmental vessel formation. PLoS One 5:e11324.

Yla-Herttuala S, Rissanen TT, Vajanto I and Hartikainen J (2007) Vascular endothelial growth factors: Biology and current status of clinical applications in cardiovascular medicine. J Am Coll Cardiol 49:1015-1026.

Zhang H, Hao J, Sun X, Zhang Y and Wei Q (2018) Circulating pro-angiogenic micro-ribonucleic acid in patients with coronary heart disease. Interact Cardiov Th 27: 336-342.

Zhu N, Zhang D, Xie H, Zhou Z, Chen H, Hu T, Bai Y, Shen Y, Yuan W, Jing Q et al. (2011) Endothelial-specific intronderived mir-126 is down-regulated in human breast cancer and targets both vegfa and pik3r2. Mol Cell Biochem 351:157-164.

\section{Supplementary Material}

The following online material is available for this article:

Table S1 - Primers for genes involved in angiogenesis in zebrafish

Table S2 - Primers for miRNAs involved in angiogenesis in HUVECs.

Associate Editor: Igor Schneider

License information: This is an open-access article distributed under the terms of the Creative Commons Attribution License (type CC-BY), which permits unrestricted use, distribution and reproduction in any medium, provided the original article is properly cited. 